\title{
Filamentary Alfvénic structures excited at the edges of equatorial plasma bubbles
}

\author{
R. Pottelette ${ }^{1}$, M. Malingre ${ }^{1}$, J. J. Berthelier ${ }^{1}$, E. Seran ${ }^{1}$, and M. Parrot ${ }^{2}$ \\ ${ }^{1}$ Centre d'Étude des Environnements Terrestre et Planétaires, CNRS, 4 av. de Neptune, 94107 St. Maur des Fossés, France \\ ${ }^{2}$ Laboratoire de Physique et Chimie de l'Environnement, 3A avenue de la Recherche Scientifique, 45071, Orléans Cedex 2, \\ France
}

Received: 25 January 2007 - Revised: 29 August 2007 - Accepted: 24 September 2007 - Published: 6 November 2007

\begin{abstract}
Recent observations performed by the French DEMETER satellite at altitudes of about $710 \mathrm{~km}$ suggest that the generation of equatorial plasma bubbles correlates with the presence of filamentary structures of field aligned currents carried by Alfvén waves. These localized structures are located at the bubble edges. We study the dynamics of the equatorial plasma bubbles, taking into account that their motion is dictated by gravity driven and displacement currents. Ion-polarization currents appear to be crucial for the accurate description of the evolution of plasma bubbles in the high altitude ionosphere. During their eastward/westward motion the bubbles intersect gravity driven currents flowing transversely with respect to the background magnetic field. The circulation of these currents is prohibited by large density depressions located at the bubble edges acting as perfect insulators. As a result, in these localized regions the transverse currents have to be locally closed by field aligned currents. Such a physical process generates kinetic Alfvén waves which appear to be stationary in the plasma bubble reference frame. Using a two-dimensional model and "in situ" wave measurements on board the DEMETER spacecraft, we give estimates for the magnitude of the field aligned currents and the associated Alfvén fields.
\end{abstract}

Keywords. Ionosphere (Ionospheric irregularities; Plasma waves and instabilities; Wave propagation)

\section{Introduction}

After sunset, the absence of sunlight in the low-latitude ionosphere leads to a much faster recombination at lower than at higher altitudes. A steep density gradient is generated between the depleted bottom side ionosphere and higher density in the upper F region. Such an anti-earthward directed

Correspondence to: R. Pottelette

(raymond.pottelette@cetp.ipsl.fr) density gradient favours the growth of a Rayleigh-Taylor ( R$\mathrm{T}$ ) instability originating at the bottom of the F layer (Hudson and Kennel, 1975). Furthermore, the gravitational field produces an ion drift and current in an eastward direction perpendicular to the magnetic field. The electrons, because of their negligible mass, do not participate in this motion. Hence, this differential drift leads to a charge separation and to the generation of electric fields inducing upward directed $\boldsymbol{E} \times \boldsymbol{B}$ drifts in depleted density-regions. The initial density rarefactions, produced at the bottom side of the equatorial ionosphere, rise up into the denser plasma of the $\mathrm{F}$ region, and this motion generates plasma bubbles. These structures are large-scale, drifting structures associated with sharp density depletions which generate turbulent spectra during their lifetime. Gradient-drift instabilities as well as turbulent cascades of long wavelength irregularities to short wavelength irregularities are usually referred to (Kelley and Mozer, 1972; Koons et al., 1997). In the present paper, we show wave data recorded by the French DEMETER (Detection of ElectroMagnetic Emissions Transmitted from Earthquake Regions) polar satellite at altitudes of $\sim 700 \mathrm{~km}$. As previously reported from the San Marco D and DE2 satellite data (Aggson et al., 1992; Aggson et al., 1996), the DEMETER data illustrate some of the characteristics of the turbulence generated by such equatorial bubbles propagating in collisionless background plasmas.

The DEMETER satellite is very well equipped for measuring waves over a wide frequency range. The electricfield instrument measures the three electric components from DC to $3.25 \mathrm{MHz}$ in four channels ULF, ELF, VLF and HF. On the other hand, the magnetic antennas measure the three magnetic components from $10 \mathrm{~Hz}$ to $20 \mathrm{kHz}$ in two channels, ELF and VLF. The present paper is focused on the interpretation of sporadic electromagnetic emissions detected at the edges of the bubbles in the ELF frequency range. These electromagnetic fluctuations occur in the regions where steep, large-scale $(1-10 \mathrm{~km})$ density gradients are recorded; they

Published by Copernicus Publications on behalf of the European Geosciences Union. 
are interpreted in the present paper in terms of Kinetic Alfvén waves. A more systematic analysis of DEMETER data reveals that such fluctuations are usually anti-correlated with broadband electrostatic emissions taking place in the lowdensity inner regions of the bubbles in conjunction with small scale $(10-100 \mathrm{~m})$ density gradients. Magnetic-field fluctuations coupled with the presence of plasma bubbles have already been reported from both the DE- 2 and the CHAMP satellite observations (Aggson et al., 1992; Luehr et al., 2002; Stolle et al., 2006). Moreover, it has been suggested that when a plasma bubble starts to grow, field aligned currents supported by Alfvén waves join the equatorial F-region with the conjugate E-regions (Bhattacharyya and Burke, 2000). The DEMETER observations seem to support the possible role played by Alfvén waves with regard to the dynamics of such equatorial plasma density depletions.

The structure of the paper is as follows. In Sect. 2 we present observations pertinent to the presence of localized, Alfvén-wave bursts at the edges of plasma bubbles. In Sect. 3, we study theoretically the excitation mechanisms of these waves from the closure by field-aligned currents of gravity-driven currents flowing transversely with respect to the background magnetic field. After some discussions, the conclusions of the paper are summarized in Sect. 4.

\section{Observations}

Figure 1 shows over a $10 \mathrm{~s}$ interval a sequence of DEMETER wave observations recorded throughout the crossing of a plasma bubble during a magnetic storm on 18 January 2005 $\left(D_{s t}=-121 \mathrm{nT}, K_{p}=8-\right)$ at 22.4 MLT (orbit 2907). DEMETER was on a poleward pass at geomagnetic latitude $\sim-2.7^{\circ}$ and altitude $\sim 700 \mathrm{~km}$. This orbit has been selected because, at the quoted times, the satellite was in a burst mode acquiring high time resolution data and providing the waveform of these electromagnetic emissions. The coordinate system used in Fig. 1 is the following: ox axis is directed eastward, oy upward and oz along the magnetic field lines. Panel (a) gives the ion density variation as measured by the onboard retarding potential analyzer (RPA); panels (b) to (g) display the three components of the magnetic field and electric fluctuations from $10 \mathrm{~Hz}$ up to $200 \mathrm{~Hz}$. The last two panels illustrate the results obtained thanks to PRASSADCO, which is a computer program used to analyse the cross-spectral matrix obtained from multi-component measurements of electromagnetic waves. It implements a number of methods to estimate polarisation and propagation parameters (Santolik, 2003). Panel (h) assesses the planarity of propagating electromagnetic waves, whereas panel (i) indicates the polar angle of propagation of the wave vector with respect to the background magnetic field.

The satellite enters into the eastern edge of the plasma bubble at about 09:04:43 UT when the density starts to decrease slightly. Two well defined electromagnetic bursts - located inside the large scale density gradient - can be identified during this time period at 09:04:45.500 UT and at 09:04:47.500 UT, respectively. According to the measurements displayed in the last two panels of Fig. 1, these bursts can be tentatively identified with kinetic Alfvén waves because they are plane waves propagating in a direction parallel to the magnetic field.

Figures $2 \mathrm{a}$ and $\mathrm{b}$ display the waveforms of the eastward $E_{x}$ electric and vertical $B_{y}$ magnetic components recorded at the times when the two electromagnetic bursts occurred. Their maximum amplitudes reach about $5 \mathrm{mV} / \mathrm{m}$ and $0.13 \mathrm{nT}$, respectively. Each burst lasts approximately $\sim 250 \mathrm{~ms}$. Using on-board DC electric field and RPA measurements the relative eastward directed velocity of the spacecraft with respect to the bubble amounts to $\sim 4 \mathrm{~km} / \mathrm{s}$, Assuming that the spacecraft crosses localized spatial structures, we get $L_{x} \sim 1 \mathrm{~km}$ for the typical perpendicular size of the bursts.

These overall observations are consistent with the presence of filamentary Alfvénic structures, located at the edge of a plasma bubble, which propagate in a direction parallel to the magnetic field lines: their typical transverse dimension is $\sim 1 \mathrm{~km}$.

Starting from 09:04:50, the density becomes very low $\left(\leq 10^{3} \mathrm{~cm}^{-3}\right)$ and intense broadband electrostatic noise emissions are recorded in association with small-scale size (in the range of 10-100 m) density structures which are detected by the on board RPA. Shortly after this time, two other magnetic bursts are recorded but their electric components are covered by the presence of a strong electrostatic turbulence generated by the existence of these small-scale density-gradients.

It is worth mentioning that these observations are quite systematic. At the DEMETER altitude, well developed plasma bubbles are generally recorded. These bubbles are all associated with bursts of low frequency electromagnetic waves taking place on the eastern and western external edges in association with steep density depression. Note, however, that the magnitude of these electromagnetic emissions may be dissimilar on these two edges. It is interesting to point out, that the electromagnetic bursts are also recorded even in the case where the bubbles are not so well developed, being associated with moderate density depressions. A preliminary data analysis reveals that, for generating such electromagnetic bursts, the density ratio between the background plasma and the internal part of the bubble has to be greater than about three.

In contrast, in the regions located at the inner edges of the bubbles, the generation of intense broadband electrostatic turbulence excited by small-scale density-gradients appears as the dominant physical process.

\section{Excitation of kinetic Alfvén waves}

In this section, frequent use is made of Fourier transforms of quantities $\boldsymbol{F}(\boldsymbol{r}, t)$ that depend both on the space coordinates 


\section{DEMETER 2005/01/18 Orbit: 02907_1}

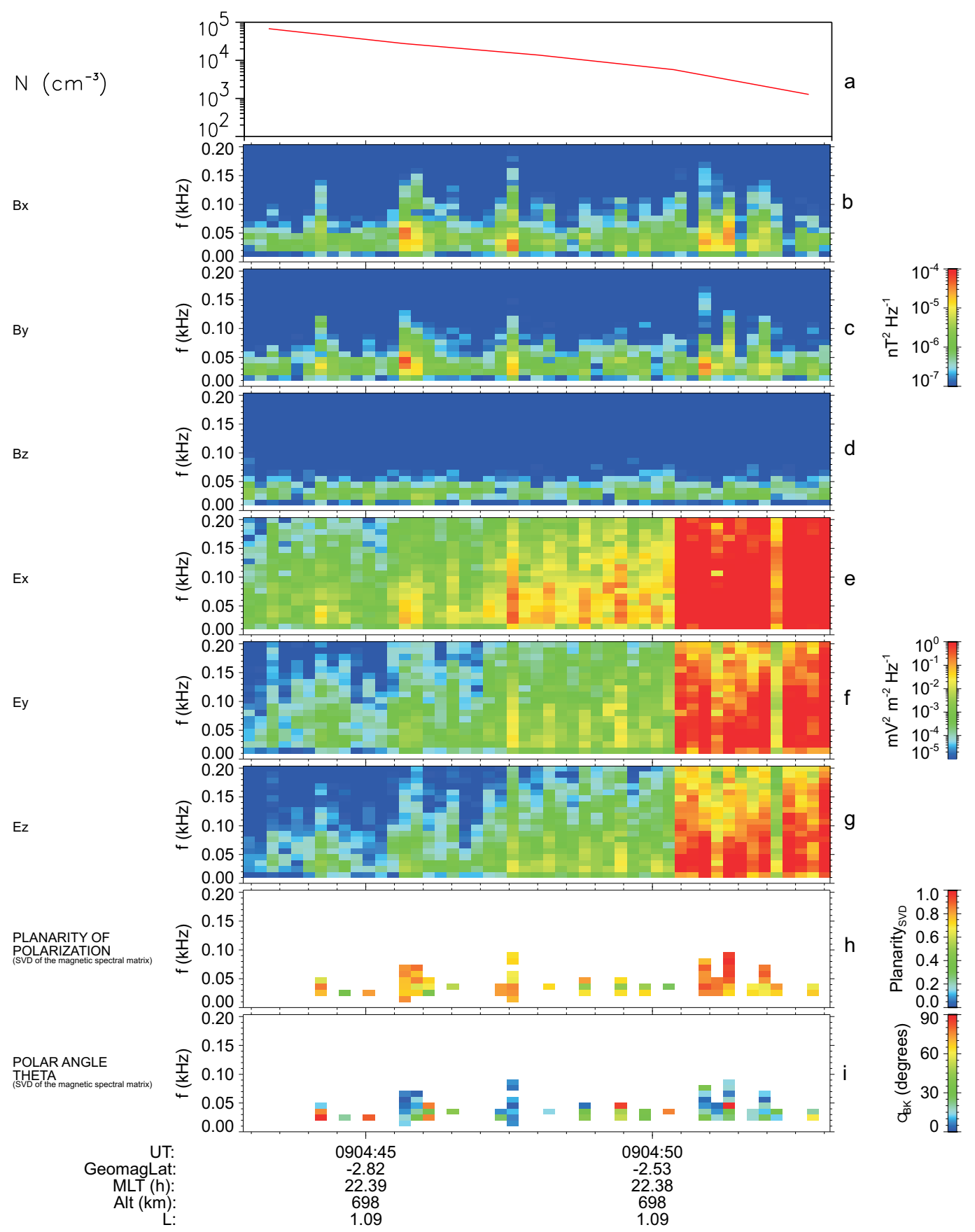

Fig. 1. The $10 \mathrm{~s}$ high resolution overview of the DEMETER crossing of a plasma bubble edges described in the text. The ox axis is directed eastward, oy upward and oz along the magnetic field lines.

$\boldsymbol{r}$ and on the time $t$. The Fourier transform is defined as

$\boldsymbol{F}(\boldsymbol{k}, \omega)=\int \boldsymbol{F}(\boldsymbol{r}, t) \operatorname{expi}(\boldsymbol{k} . \boldsymbol{r}-\omega t) d r d t$

where $\omega$ is the angular frequency and $\boldsymbol{k}$ is the wave number vector.
Conversely, the inverse Fourier transform is given by

$$
\boldsymbol{F}(\boldsymbol{r}, t)=\frac{1}{(2 \pi)^{4}} \int \boldsymbol{F}(\boldsymbol{k}, \omega) \exp -i(\boldsymbol{k} \cdot \boldsymbol{r}-\omega t) d \boldsymbol{k} d \omega
$$

With this convention, the spatio-temporal Fourier transform of the unit plane wave $\boldsymbol{F}(\boldsymbol{r}, \mathrm{t})=\exp \left[\mathrm{i}\left(\omega t-\boldsymbol{k}_{0} . \boldsymbol{r}\right)\right]$ is the Dirac distribution $\boldsymbol{F}(\boldsymbol{k}, \omega)=(2 \pi)^{4} \delta\left(\boldsymbol{k}-\boldsymbol{k}_{0}\right) \delta\left(\omega-\omega_{0}\right)$. 

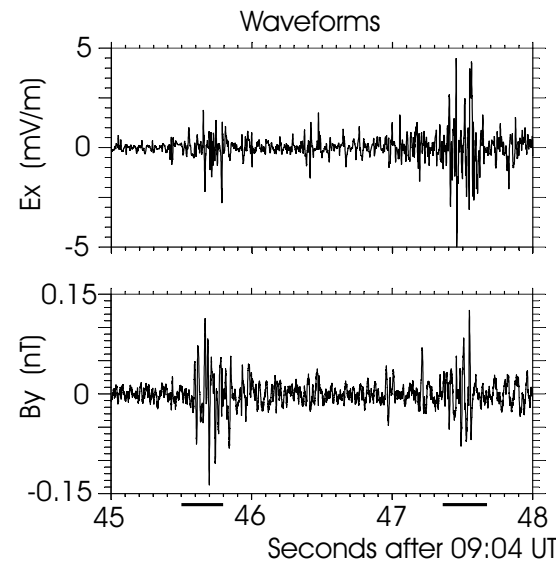

a

b
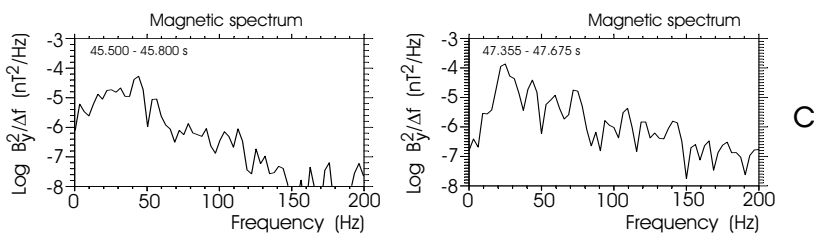

Fig. 2. Illustration of the wave data recorded during the occurrence of two well-defined electromagnetic bursts: (a) Waveform of the $E_{x}$ electric field component, (b) Waveform of the $B_{y}$ magnetic component, (c) Magnetic spectra showing well defined maxima at $\sim 40 \mathrm{~Hz}$ and at $\sim 25 \mathrm{~Hz}$.

Although bubbles are three-dimensional entities, we approximate them as two-dimensional structures for the sake of simplicity. We use an $\mathrm{x}-\mathrm{Z}$ coordinate system whose center $\mathrm{O}$ is located at the satellite: the $\mathrm{x}$ axis is oriented in the eastward direction while the $\mathrm{z}$ axis is oriented along the earth's magnetic field direction. All physical quantities are assumed to be uniform along the y vertical axis so that $\partial / \partial y=0$. The eastward current density $j_{x}$ can be expressed as the sum of the gravity-driven current density $j_{g}$ and of the ion polarization current density induced by the low frequency electric fluctuations $E_{x}$. We get

$j_{x}=\frac{1}{\mu_{0} V_{A}^{2}} \partial_{t} E_{x}+j_{g}$

where $V_{A}$ is the Alfvén velocity, while $j_{g}=n m_{i} g / B_{0}$ can be expressed in terms of the ion density $n$, ion mass $m_{i}$, and $g$ is gravity. Ion polarization currents appear to be crucial for the accurate description of the evolution of plasma bubbles in the high altitude ionosphere (Keskinen et al., 1998).

In the plasma, the electrons are magnetized and move only parallel to the magnetic field lines $\mathbf{B}_{0}=B_{0} \mathbf{z}$ in order to close the transverse currents. Using the momentum transfer equation, the parallel current density is given by

$\partial_{t} j_{z}+\partial_{z}\left(\frac{j_{z}^{2}}{n e}\right)=\frac{n e^{2}}{m_{e}} E_{z}$ where $e$ is the electron charge, $m_{e}$ the electron mass, and $\mathrm{n}$ the electron density (assumed equal to the ion density).

In addition, the Maxwell equations can be written as:

$\nabla x(\nabla x \boldsymbol{E})=-\mu_{0} \partial_{t} \boldsymbol{j}$

The plasma is then described by the system of Eqs. (3-5). Linearizing these equations, after Fourier transformation for the perpendicular electric field, we find

$E_{x}(\omega, k)=\frac{-i}{\varepsilon_{0}}\left(\frac{V_{A}}{c}\right)^{2} \omega\left[\omega^{2}-k_{z}^{2} V_{A}^{2} /\left(1+k_{x}^{2} c^{2} / \omega_{P}^{2}\right)\right]^{-1} j_{g}(\omega, k)(6)$

where $\varepsilon_{0}$ is the electric permittivity of free space, $c$ the speed of light, and $\omega_{p}$ the angular plasma frequency. The expression in brackets is the well-known dispersion relation of kinetic (shear) Alfvén waves in the low $\beta$ approximation $\left(\beta<m_{e} / m_{i}\right)$, where $\beta$ is the ratio of thermal-to-magnetic energy. This approximation is fulfilled, as the Alfvén speed $V_{A}$ is larger than the electron thermal velocity $V_{t e}$ of the cold background plasma. Equation (6) shows that gravity-driven current perturbations generate Alfvén electromagnetic fluctuations.

As already pointed out, the gravity driven current being proportional to the ion density is smaller within the plasma bubble than outside it. As a result charges pile up at the edges of the plasma bubble producing an eastward electric field within the bubble, which makes the bubble moves upward. Using Eq. (6), it is possible to calculate, on the edge of a plasma bubble, the electric field fluctuations $E_{x}(\mathrm{x}, \mathrm{z}, \mathrm{t})$ resulting from the large variations $\delta j_{g}(\mathbf{r}, \mathrm{t})$ in the gravity-driven current. In these boundary regions, $\delta j_{g}(\mathbf{r}, \mathrm{t})$ can be modeled by a Gaussian distribution to allow for easy calculations

$\delta j_{g}(\boldsymbol{r}, t)=\boldsymbol{e}_{x} j_{0}\left\{1-\exp -\left[\frac{\left(x-U_{x} t\right)^{2}}{\Delta_{x}^{2}}+\frac{z^{2}}{\Delta_{z}^{2}}\right]\right\}$

where $j_{0}=n m_{i} g / B_{0}$ is the magnitude of the gravity driven current density flowing in the background plasma previously defined. The electric field fluctuations being measured in the satellite reference frame, $U_{x}$ denotes the magnitude of the eastward component of the relative speed of a bubble with respect to the satellite. The Gaussian distribution has a width $\Delta_{x}$ in the $\mathrm{x}$ direction, $\Delta_{z}$ in the $\mathrm{z}$ direction and is assumed to be infinite in the y direction.

From Eq. (1) we obtain $\delta j_{g}(\mathbf{k}, \omega)=\delta j_{g 1}(\mathbf{k}, \omega)+\delta j_{g 2}(\mathbf{k}, \omega)$ with $\delta j_{g 1}(\mathbf{k}, \omega)=(2 \pi)^{4} j_{0} \delta(\omega) \delta(\mathbf{k})$, and

$$
\begin{aligned}
\delta j_{g 2}(\boldsymbol{k}, \omega)= & -4 \pi^{3} j_{0} \Delta_{x} \Delta_{z} \delta\left(\omega-k_{x} U_{x}\right) \delta\left(k_{y}\right) \\
& \exp -\left[\left(\frac{k_{x} \Delta_{x}}{2}\right)^{2}+\left(\frac{k_{z} \Delta_{z}}{2}\right)^{2}\right]
\end{aligned}
$$

In order to calculate the space and time electric field fluctuations we have to insert the expression for $\delta \mathbf{j}_{g}(\mathbf{k}, \omega)$ in Eq. (6) and to take the inverse Fourier transform. Note that the term 
$\delta j_{g 1}(\mathbf{k}, \omega)$ does not bring any contribution to the integral. After some lengthy but straightforward calculations, we get

$$
\begin{aligned}
E_{x}(x, z, t)= & \frac{\Delta_{x} \Delta_{z}}{\varepsilon_{0}} j_{0} \frac{V_{A}}{c^{2}}\left(\frac{\pi}{4 \chi}\right)^{1 / 2} \\
& \exp \left[-\left(\frac{\left(x-U_{x} t\right)^{2}+\alpha^{2} z^{2}}{4 \chi}\right)\right] \\
& \cosh \left(\frac{\left(x-U_{x} t\right) \alpha z}{2 \chi}\right)
\end{aligned}
$$

where $\alpha=U_{x} / V_{A}$ and $\chi=\left[\Delta_{x}^{2}+\left(\alpha \Delta_{z}\right)^{2}\right] / 4$. In the background equatorial ionospheric plasma, we usually have $U_{x} \sim 1 \mathrm{~km} / \mathrm{s}$ and $V_{A} \sim 1000 \mathrm{~km} / \mathrm{s}$. Accordingly, the relative drift velocity of a bubble with respect to the satellite is much smaller than the background Alfvén velocity and $\alpha \ll 1$. As a consequence, in the frame moving with the bubble, the excited electric field component of the Alfvén wave is a quasistationary structure varying slowly along $\mathrm{z}$, its spatial spread is $2(\chi)^{1 / 2}$. Note that $V_{A}$ is the local value of the Alfvén velocity at a selected location. The associated magnetic component is such that $B_{y}=E_{x} / V_{A}$.

Let us take the example of orbit 2907. Characteristic values measured by the DEMETER spacecraft during this plasma bubble event are: $U_{x} \sim 4 \mathrm{~km} / \mathrm{s}$ (as previously defined), and $B \sim 2.7 \times 10^{4} \mathrm{nT}$. At the edge of the bubble, the half-width of the depleted region is traversed in about $2.5 \mathrm{~s}$ leading to $\Delta_{x} \sim 10 \mathrm{~km}$ (see Fig. 3). During this event, the satellite traverses the bubble in a predominantly south-north oriented direction, with a velocity of $\sim 6 \mathrm{~km} / \mathrm{s}$ in this direction. The associated density depression is recorded during $\sim 3 \mathrm{~min}$, leading to $\Delta_{z} \sim 1000 \mathrm{~km}$.

The density of the background plasma outside the bubble at the satellite altitude is $\sim 10^{5} \mathrm{~cm}^{-3}$ implying to $j_{0} \sim 10^{-9} \mathrm{~A} / \mathrm{m}^{2}$ for an $\mathrm{O}^{+}$dominated plasma. The measured background electron temperature is $0.2 \mathrm{eV}$ which matches with an electron thermal velocity $V_{t e} \sim 180 \mathrm{~km} / \mathrm{s}$, so that the inequality $V_{t e}<V_{A}$ is fulfilled. At the location where the first electromagnetic burst is recorded by DEMETER (at 09:04:45.500 UT) the density has decreased to $\sim 5.10^{4} \mathrm{~cm}^{-3}$ corresponding to $V_{A} \sim 700 \mathrm{~km} / \mathrm{s}$ for the velocity of the propagating Alfvén waves. At the second burst location (occurring around 09:04:47.000 UT) the density has dropped to $2.4 \times 10^{4} \mathrm{~cm}^{-3}$ so that $V_{A} \sim 1000 \mathrm{~km} / \mathrm{s}$. Using Eq. (9) with these quoted values, we get $\sim 2 \mathrm{mV} / \mathrm{m}$ for the amplitude of the $E_{x}$ electric field fluctuations at the satellite altitude. This value corresponds to a correct order of magnitude with respect to the measured values (see Fig. 2a). Such a reasonable agreement with experimental values seems satisfactory in view of the over simplified theory used in this paper.

More generally speaking, a careful analysis of DEMETER data reveals that typical values for the amplitudes of electromagnetic bursts recorded by the DEMETER spacecraft are in the range $3-10 \mathrm{mV} / \mathrm{m}$ for the electric component and $0.1-0.3 \mathrm{nT}$ for the magnetic component. The major components of the electric and magnetic perturbations are eastward

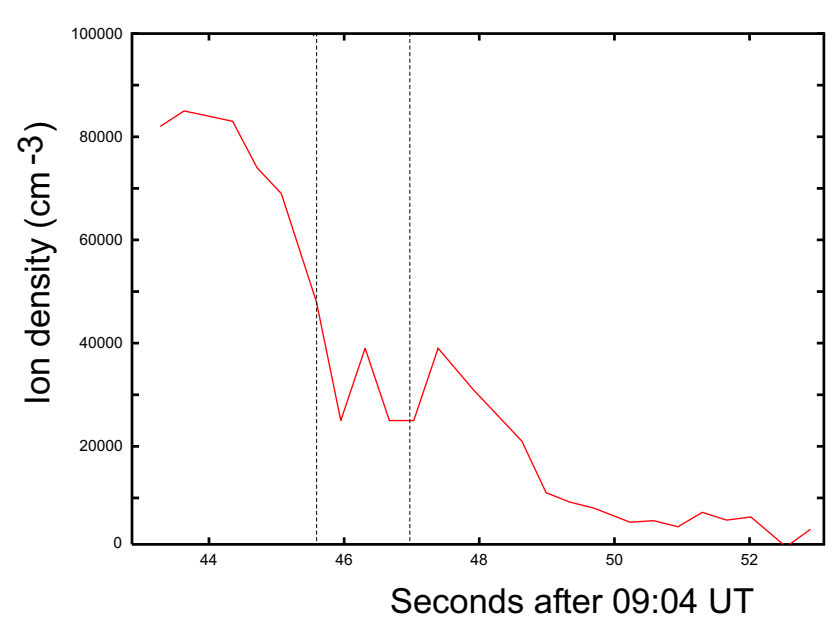

Fig. 3. High time resolution measurements of the ion density performed by the RPA as function of time. The dotted lines indicate the locations of the two investigated electromagnetic bursts.

and radially outward, respectively. The Poynting flux associated with these Alfvén waves is thus directed along the magnetic field lines. Using $10 \mathrm{mV} / \mathrm{m}$ and $0.3 \mathrm{nT}$ leads to $2.5 \times 10^{-6} \mathrm{~W} / \mathrm{m}^{2}$ for the peak Poynting flux.

As derived from Eqs. (6) and (8), in the $\omega, k$ domain $E_{x}$ and $B_{y}$ should be rather monochromatic with an angular frequency $\omega=k_{x} U_{x}$ and a perpendicular wave-length with a spectral width $\delta k_{x} \sim 1 / \Delta_{x}$. Furthermore, it is well known that for a kinetic (shear) Alfvén wave $k_{x} \sim \omega_{p} / c$, this leads to $\omega \sim U_{x} \omega_{p} / c$. A more accurate evaluation of $k_{x}$ can be derived from a careful analysis of the characteristics of the electromagnetic fluctuations.

The spectra of the magnetic fluctuations $B_{y}$ recorded in the satellite reference frame at the locations of the two electromagnetic bursts are displayed in Fig. 2c. In this frame the transverse drift velocity of the bubble with respect to DEMETER amounts to $U_{x} \sim 4 \mathrm{~km} / \mathrm{s}$. As shown in Fig. $2 \mathrm{c}$, the magnetic spectra peak at frequencies around $f_{1} \sim 40 \mathrm{~Hz}$ and $f_{2} \sim 25 \mathrm{~Hz}$, respectively, and are such that $f_{1} / f_{2} \sim f_{p 1} / f_{p 2}$ where $f p_{1}=2 \mathrm{MHz}$ and $f p_{2}=1.4 \mathrm{MHz}$ are the respective plasma frequencies at the locations where the two electromagnetic burst are recorded. As a result, the frequencies for which the magnetic spectra maximize are well approximated by $f=3 U_{x} f_{p} / 2 c$. In the angular frequency domain we thus have $\omega=k_{x} U_{x}$ with $k_{x}=3 \omega_{p} / 2 c$.

For the first burst (around 09:04:45.500 UT), we get $k_{x}=6.3 \times 10^{-2} \mathrm{~m}^{-1}$ corresponding to a perpendicular wavelength $\lambda_{x}=100 \mathrm{~m}$, while for the second burst we get $k_{x}=4.4 \times 10^{-2} \mathrm{~m}^{-1}$ and $\lambda_{x}=140 \mathrm{~m}$. The perpendicular size of the electromagnetic pulses previously derived $\left(L_{x} \sim 1 \mathrm{~km}\right)$ matches respectively with 10 or 7 field oscillations, in reasonable agreement with the observations (see Fig. 2). The broadband character of the spectrum should be a consequence of the plasma inhomogeneities which are not properly taken into account in the present theory. 
The field aligned current density $j_{z}$ connected with the kinetic Alfvén wave (Lysak and Carlson, 1981) can be obtained from:

$j_{z}=\left(\frac{\varepsilon_{0} c^{2}}{V_{A}}\right) \partial_{x} E_{x}$

Taking into account that $\alpha \ll 1$, we get:

$$
\begin{aligned}
j_{z}= & \frac{\Delta_{x} \Delta_{z}}{4} j_{0}\left(\frac{\pi}{\chi^{3}}\right)^{1 / 2}\left(x-U_{x} t\right) \\
& \exp \left[-\left(\frac{\left(x-U_{x} t\right)^{2}+\alpha^{2} z^{2}}{4 \chi}\right)\right] \\
& \cosh \left(\frac{\left(x-U_{x} t\right) \alpha z}{2 \chi}\right)
\end{aligned}
$$

In the bubble reference frame, the current density is the strongest at $\partial_{(x-U x t)} j_{z}=0$, which leads to $\left(x-U_{x} t\right) \sim(2 \chi)^{1 / 2}$. This shows that the gravity driven currents are closed by field aligned currents flowing at the bubble edges. This estimate agrees fairly well with the first burst location but not with that of the second burst. This discrepancy may be understood from the high time resolution data of the plasma density displayed in Fig. 3. It appears that the second burst is generated inside a well defined density structure of about $1 \mathrm{~km}$ transverse scale which traps the Alfvén waves, thereby enhancing locally their amplitude. These observations bring to light the role of small scale density structures in generating turbulent spectra at the edges of plasma bubbles. Similar observations have been performed in the auroral ionosphere (Stasiewicz et al., 2000).

The maximum value of the current density is obtained for $\left(x-U_{x} t\right)=(2 \chi)^{1 / 2}$; this leads to:

$j_{z}^{\max } \sim(2 \pi)^{1 / 2} \frac{\Delta_{x} \Delta_{z}}{4 \chi} j_{0} \exp (-1 / 2)$

With the plasma parameters previously defined, we get $j_{z}^{\max }=0.2 \mu \mathrm{A} / \mathrm{m}^{2}$. Unfortunately, there was no scientific instrument to measure DC magnetic fields on DEMETER, the flux gate is simply an attitude magnetometer with a poor sensitivity $\left(\sim 0.5 \mu \mathrm{A} / \mathrm{m}^{2}\right)$. Consequently, the magnitude of such currents cannot be measured. The field aligned currents should be carried by suprathermal electrons drifting in resonance with the propagating Alfvén waves. The parallel drift velocity of these resonant electrons should be in the range of $\sim 1000 \mathrm{~km} / \mathrm{s}$, this corresponds to an energy of $\sim 1.5 \mathrm{eV}$. They should form filamentary structures which may locally act as an additional source of electrostatic turbulence by generating waves instabilities in the neighbourhood of the $\mathrm{O}^{+}$ion cyclotron frequency (Kindel and Kennel, 1971).

\section{Conclusions}

A number of night-time DEMETER orbits have provided new insights into the highly disturbed equatorial ionosphere. In the present paper, we have shown that during these disturbed time-periods, the edges of equatorial densitydepressions are characterized by the presence of filamentary structures with a typical kilometre transverse width. Preliminary data analysis reveals that such structures are usually generated as long as the density of the background plasma overcomes the density inside the bubble by at least a factor three.

Inside these structures, propagating kinetic Alfvén waves carrying field aligned currents have been identified. We have pointed out that the excitation of these waves might be generated by large density depletions which affect the gravitydriven currents collected by the bubbles during their eastward/westward motion. Due to the large surface area covered by the bubbles the amount of collected gravity currents is not negligible in spite of the weakness of these currents. The present observations are consistent with the role of Alfvén waves in connecting the equatorial $\mathrm{F}$ region and the conjugate E regions (Aggson et al., 1992; Bhattacharyya and Burke, 2000). It is worth mentioning that these waves influence the dynamics of a plasma bubble by providing, in the bubble reference frame, an additional quasi-static electric field component transverse to the magnetic field.

Thanks to the relative motion bubbles/spacecraft, the low frequency Alfvénic electromagnetic turbulence is up shifted in the ELF frequency range. The most useful information provided by the simple theory developed in this paper deals with a correct estimate for the amplitude of these frequency shifts. In addition, the turbulent spectra are broadened by the existence of plasma inhomogeneities which are not properly taken into account in the present overly simplified theory. It should be stressed, however, that at the external edges of the equatorial density-depletions, the density-gradient scales $\Delta_{x}$ are usually much larger than the derived values of the Alfvén perpendicular wavelengths $\lambda_{x}$, so that locally the main characteristics of these waves should not be too much distorted.

Acknowledgements. The authors thank the CNES and industry teams who took part in the DEMETER program for their dedication and achievements in designing, building and integrating the satellite. The authors also thank A. Bhattacharyya (IIGM, India), R. Pfaff ( GSSL, USA) and M. Berthomier (CETP/CNRS, France) for useful discussions on the present subject.

Topical Editor M. Pinnock thanks J. Makela and another anonymous referee for their help in evaluating this paper.

\section{References}

Aggson, T. L., Burke, W. J., Maynard, N. C., Hanson, W. B., Anderson, P. C., Slavin, J. A., Hoegy, W. R., and Saba, J. L.: Equatorial bubbles up drafting at supersonic speeds, J. Geophys. Res., 97, 8581-8590, 1992.

Aggson, T. L., Laakso, H., Maynard, N. C., and Pfaff, R. F.: In situ observations of bifurcation of equatorial ionospheric plasma depletions, J. Geophys. Res., 101, 5125-5133, 1996. 
Bhattacharyya, A. and Burke, W. J.: A transmission line analogy for the development of equatorial ionospheric bubbles, J. Geophys. Res., 105, 24 941-24 950, 2000.

Hudson, M. K. and Kennel, C. F.: Linear Theory of equatorial spread F, J. Geophys. Res., 80, 4581-4590, 1975.

Kelley, M. C. and Mozer, F. S.: A satellite survey of vector electric fields in the ionosphere at frequencies of 10 to 500 Hertz, low frequency equatorial emissions and their relationship to ionospheric turbulence, J. Geophys. Res., 77, 4183-4189, 1972.

Keskinen, M. J., Ossakow, S. L., Basu, S., and Sultan, P. J.: Magnetic-flux-integrated evolution of equatorial ionospheric plasma bubbles, J. Geophys. Res., 103, 3957-3967, 1998.

Kindel, J. M. and Kennel, C. F.: Topside current instabilities, J. Geophys. Res., 76, 3055-3078, 1971.

Koons, H. C., Roeder J. L., and Rodriguez, P.: Plasma waves observed inside plasma bubbles in the equatorial $\mathrm{F}$ region, J. Geophys. Res., 102, 4577-4583, 1997.
Lühr, H., Maus, S., Rother, M., and Cooke, D.: First in-situ observations of night-time $F$ region currents with the CHAMP satellite, Geophys. Res. Lett., 29, 1489-1493, doi:10.1029/2001GL013845, 2002.

Lysak, R. L. and Carlson, C. W.: The effect of microscopic turbulence on magnetosphere-ionosphere coupling, Geophys. Res. Lett., 8, 269-273, 1981.

Santolik, O.: Propagation analysis of Staff-SA data with coherency tests (A user's guide to PRASSADCO), LPCE/NTS/073.D, Lab. Phys. Chimie Environ./CNRS, Orléans, France, 2003.

Stasiewicz, K., Khotyaintsev, Y., Berthomier, M., and Walhund, J. E.: Identification of Widespread Turbulence of Dispersive Alfvén waves, Geophys. Res. Lett., 27, 173-177, 2000.

Stolle, C., Lühr, H., Rother, M., and Balasis, G.: Magnetic signatures of equatorial spread $\mathrm{F}$ as observed by the CHAMP satellite, J. Geophys. Res., 111(A2), A02304, doi:10.1029/2005JA011184, 2006. 